\title{
First measurement of the ${ }^{2} \mathrm{H}\left({ }^{6} \mathrm{He},{ }^{7} \mathrm{Li}\right) \mathrm{n}$ angular distribution and proton spectroscopic factor in ${ }^{7} \mathrm{Li}$
}

\author{
Z.H. Li ${ }^{a}$, E.T. Li, B. Guo, X.X. Bai, Y.J. Li, S.Q. Yan, Y.B. Wang, G. Lian, J. Su, B.X. Wang, S. Zeng, X. Fang, \\ and W.P. Liu
}

China Institute of Atomic Energy, P. O. Box 275(46), Beijing 102 413, PRC

\author{
Received: 29 September 2009 / Revised: 24 December 2009 \\ Published online: 6 March 2010 \\ (C) The Author(s) 2010. This article is published with open access at Springerlink.com \\ Communicated by N. Alamanos
}

\begin{abstract}
The angular distribution of the ${ }^{2} \mathrm{H}\left({ }^{6} \mathrm{He},{ }^{7} \mathrm{Li}\right) n$ reaction was measured with a secondary ${ }^{6} \mathrm{He}$ beam of $36.4 \mathrm{MeV}$ for the first time. The proton spectroscopic factor of ${ }^{7} \mathrm{Li}$ ground state was extracted to be $0.42 \pm 0.06$ by normalizing the calculational differential cross-sections with the distorted-wave Born approximation to the experimental data. It was discussed that the uncertainty of extracted spectroscopic factors from the one-nucleon transfer reactions induced by deuteron might be reduced by determining the volume integrals of imaginary optical potentials precisely.
\end{abstract}

\section{Introduction}

The essential constituents of nuclear shell model are the single-particle orbits of the mean field which are occupied by protons and neutrons under Pauli principle. The spectroscopic factor describes the overlap between the initial and final states and yields the information on the occupancy of a given single-particle orbit, which plays an important role in a variety of topics on nuclear reaction and nuclear astrophysics. Single-nucleon transfer reactions such as $(d, p)$ and $(d, n)$ have been used extensively to extract the spectroscopic information of the single-nucleon orbits in nuclei located at or near the stability line [1-3]. The spectroscopic study of exotic nuclei becomes feasible since the production of radioactive ion beams $[4-6]$. These measurements allow the extraction of the spectroscopic factors by normalizing the calculational differential cross-sections with the distorted-wave Born approximation (DWBA) to the experimental ones at forward angles.

The $\left({ }^{7} \mathrm{Li},{ }^{6} \mathrm{He}\right)$ reaction is a valuable spectroscopic tool in the study of nuclear reactions because the shape of its angular distribution can be well reproduced by DWBA calculations [7]. In the calculations of $\left({ }^{7} \mathrm{Li},{ }^{6} \mathrm{He}\right)$ reactions [7-11], the spectroscopic factor of the ${ }^{7} \mathrm{Li}$ ground state was taken to be 0.59 [12]. Brady et al. [13] extracted the spectroscopic factor of the ${ }^{7} \mathrm{Li}$ ground state to be $S\left(p_{3 / 2}\right)=0.62$ from the ${ }^{7} \operatorname{Li}(n, d){ }^{6} \mathrm{He}$ reaction with $56.3 \mathrm{MeV}$ neutrons. Lapikás et al. [14] deduced the proton spectroscopic factor of ${ }^{7} \mathrm{Li}$ to be $0.42 \pm 0.04$ via the measurement of the ${ }^{7} \mathrm{Li}\left(e, e^{\prime} p\right)$ reaction. This value is $32 \%$

\footnotetext{
a e-mail: zhli@ciae.ac.cn
}

smaller than that from the ${ }^{7} \operatorname{Li}(n, d){ }^{6} \mathrm{He}$ reaction. Thus, further measurement of the ${ }^{7} \mathrm{Li}$ spectroscopic factor is highly desired.

In the present work, the ${ }^{2} \mathrm{H}\left({ }^{6} \mathrm{He},{ }^{7} \mathrm{Li}\right) n$ angular distribution was measured by using a secondary ${ }^{6} \mathrm{He}$ beam of $36.4 \mathrm{MeV}$ and analyzed with DWBA. The proton spectroscopic factor in ${ }^{7} \mathrm{Li}$ was then extracted and compared with the existing ones.

\section{Measurement of the angular distribution}

The experiment was carried out using the secondary beam facility [15] of the HI-13 tandem accelerator, Beijing. A $46 \mathrm{MeV}^{7} \mathrm{Li}$ primary beam from the tandem impinged on a $4.8 \mathrm{~cm}$ long deuterium gas cell at a pressure of about $1.5 \mathrm{~atm}$. The front and rear windows of the gas cell are Havar foils, each with thickness of $1.9 \mathrm{mg} / \mathrm{cm}^{2}$. The ${ }^{6} \mathrm{He}$ ions were produced via the ${ }^{2} \mathrm{H}\left({ }^{7} \mathrm{Li},{ }^{6} \mathrm{He}\right){ }^{3} \mathrm{He}$ reaction. After the magnetic separation and focus with a dipole and a quadruple doublet, a $37.6 \mathrm{MeV}$ secondary ${ }^{6} \mathrm{He}$ beam was delivered and then collimated with a $\varnothing 7-\varnothing 5 \mathrm{~mm}$ collimator complex. The ${ }^{6} \mathrm{He}$ beam was then recorded by a $23 \mu \mathrm{m}$ thick silicon $\Delta E_{1}$ detector, which served as both particle identification and beam normalization. The typical purity and intensity of the ${ }^{6} \mathrm{He}$ beam are, respectively, $99 \%$ and $3000 \mathrm{pps}$. The main contaminants were ${ }^{7} \mathrm{Li}$ ions out of Rutherford scattering of the primary beam in the gas cell windows as well as on the beam tube, which can be excluded in the data analysis by the selection of ${ }^{6} \mathrm{He}$ peak in the $\Delta E_{1}$ spectrum. 


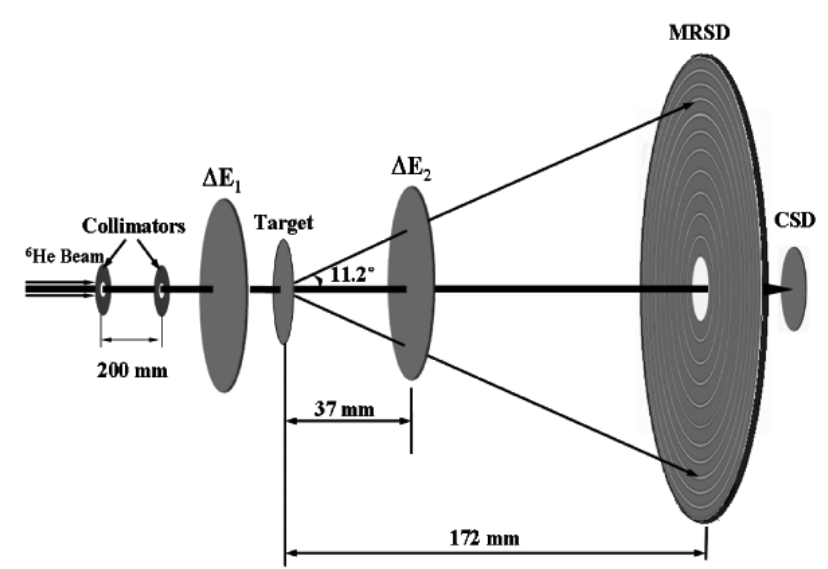

Fig. 1. Schematic layout of the experimental setup.

The experimental setup is shown in fig. 1. A $\left(\mathrm{CD}_{2}\right)_{n}$ foil and a carbon foil, both with thickness of $1.7 \mathrm{mg} / \mathrm{cm}^{2}$, were used as the targets to measure the ${ }^{2} \mathrm{H}\left({ }^{6} \mathrm{He},{ }^{7} \mathrm{Li}\right) n$ reaction and background, respectively. The energy of ${ }^{6} \mathrm{He}$ ions at the middle of the $\left(\mathrm{CD}_{2}\right)_{n}$ target was $36.4 \mathrm{MeV}$. A $300 \mu \mathrm{m}$ thick multi-ring semiconductor detector (MRSD) with center hole was used as a residue energy $\left(E_{r}\right)$ detector which composed a $\Delta E-E_{r}$ counter telescope with a $23 \mu \mathrm{m}$ thick silicon $\Delta E_{2}$ detector and a $300 \mu \mathrm{m}$ thick center silicon detector (CSD). CSD was mainly used to monitor the purity of the ${ }^{6} \mathrm{He}$ beam together with the $\Delta E_{2}$ detector. Such a detector configuration covered the laboratory angular range from $0^{\circ}$ to $11.2^{\circ}$, and the corresponding angular range in the center-of-mass frame for the ${ }^{2} \mathrm{H}\left({ }^{6} \mathrm{He},{ }^{7} \mathrm{Li}\right) n$ reaction was from $0^{\circ}$ to $51.6^{\circ}$. Generally, the spectroscopic factor is extracted by fitting the theoretical calculations to the experimental data at the first peak in the angular distribution at forward angles [16], since the experimental angular distribution at the backward angles is more sensitive to the inelastic coupling effects and other high-order ones, which cannot be well described theoretically. The DWBA calculation predicts that the first peak of the angular distribution for the ${ }^{2} \mathrm{H}\left({ }^{6} \mathrm{He},{ }^{7} \mathrm{Li}\right) n$ reaction is around $20^{\circ}$ in the center-of-mass frame, thus the present setup is propitious to the extraction of the ${ }^{7} \mathrm{Li}$ spectroscopic factor.

The accumulated quantity of incident ${ }^{6} \mathrm{He}$ was approximately $2.71 \times 10^{8}$ for the $\left(\mathrm{CD}_{2}\right)_{n}$ target measurement, and $8.41 \times 10^{7}$ for background measurement with the carbon target. As an example, fig. 2 displays the $\Delta E-E_{r}$ scatter plots of both $\left(\mathrm{CD}_{2}\right)_{n}$ and carbon targets for the fourth ring of MRSD. For the sake of saving CPU time in dealing with the experimental data, we set a cut at $\Delta E=2.0 \mathrm{MeV}$. All the events below the cut were scaled down by a factor of 100 , while the ${ }^{7} \mathrm{Li}$ events remained unchanged. The solid curves in fig. 2 are the calculated $\Delta E$ vs. $E_{r}$ for ${ }^{7} \mathrm{Li},{ }^{6} \mathrm{Li}$ and ${ }^{6} \mathrm{He}$, respectively. The twodimensional gates with dashed circles are the ${ }^{7} \mathrm{Li}$ kinematics regions from the ${ }^{2} \mathrm{H}\left({ }^{6} \mathrm{He},{ }^{7} \mathrm{Li}\right) n$ reaction, corresponding to the fourth ring. The ${ }^{7} \mathrm{Li}$ events can be clearly identified in this figure. We did not find any ${ }^{7} \mathrm{Li}$ event in the gate for the background runs. Figure 3 shows the scatter plot of total energy $\left(E_{t}\right)$ vs. laboratory angles $\left(\theta_{\text {lab }}\right)$ for the

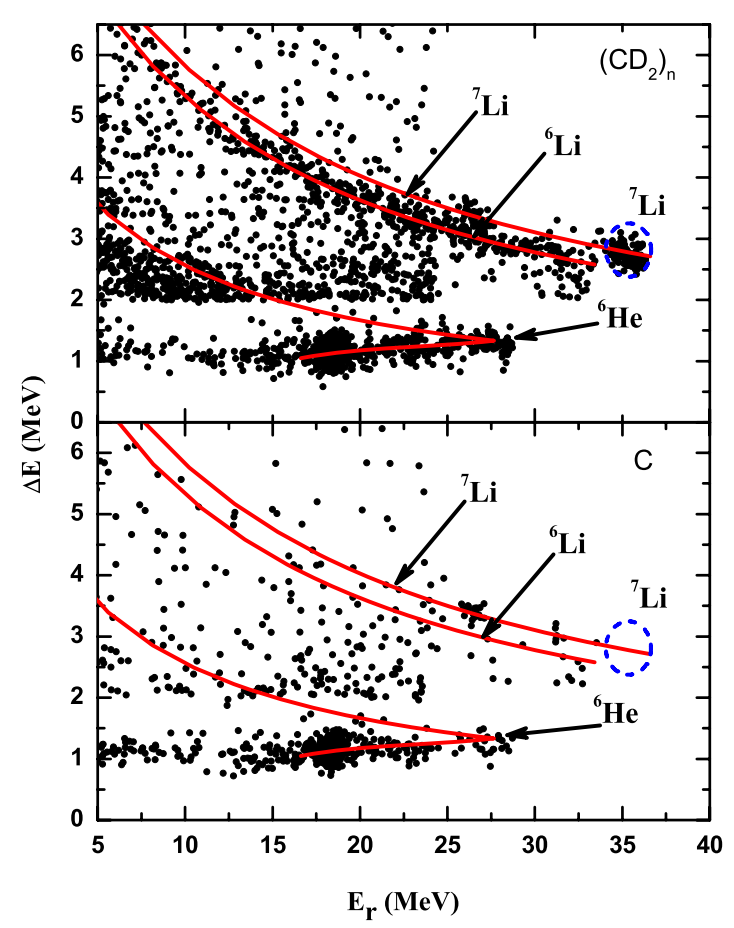

Fig. 2. $\Delta E$ vs. $E_{r}$ scatter plots of $\left(\mathrm{CD}_{2}\right)_{n}$ target (top panel) and pure carbon target (bottom panel) measured by the fourth ring of MRSD. The solid curves are the calculational $\Delta E v s$. $E_{r}$ for the particle identification of ${ }^{7} \mathrm{Li},{ }^{6} \mathrm{Li}$ and ${ }^{6} \mathrm{He}$. The twodimensional gates with dashed circles are the ${ }^{7} \mathrm{Li}$ kinematics regions from the ${ }^{2} \mathrm{H}\left({ }^{6} \mathrm{He},{ }^{7} \mathrm{Li}\right) n$ reaction, corresponding to the fourth ring $\left(\theta_{\text {c.m. }}=25.8^{\circ}-30.9^{\circ}\right)$.

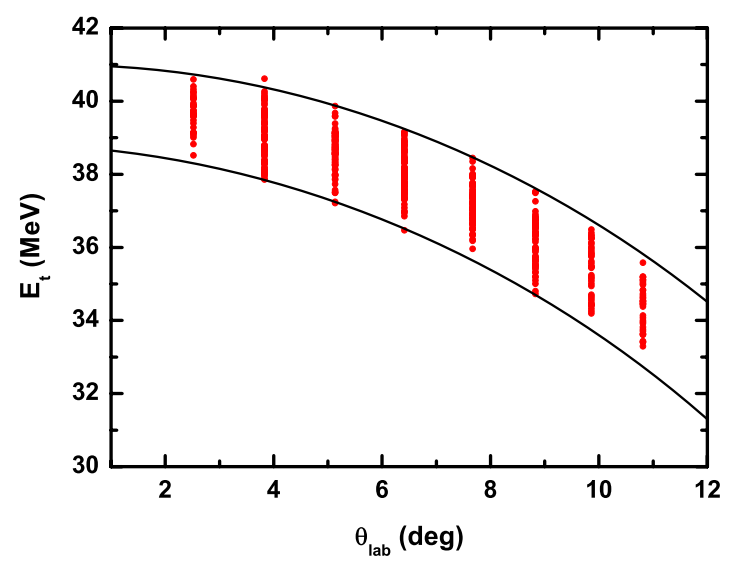

Fig. 3. Scatter plot of $E_{t}$ vs. $\theta_{\text {lab }}$ for the events within the ${ }^{7} \mathrm{Li}$ two-dimensional gate in fig. 2 .

events within the two-dimensional gate of ${ }^{7} \mathrm{Li}$ kinematics region. The zone between the two solid lines represents the kinematics region for both the ground and excited states of ${ }^{7} \mathrm{Li}$, based on a Monte Carlo simulation. The simulation took into account the beam spot size, energy spread, angular divergence, and target thickness. The measured angular distribution is shown in fig. 5. The uncertainties of differential cross-sections mainly arose from the statistics and the assignment of ${ }^{7} \mathrm{Li}$ kinematics regions. The angular error was given by the Monte Carlo simulation. 


\section{DWBA calculations}

The ${ }^{2} \mathrm{H}\left({ }^{6} \mathrm{He},{ }^{7} \mathrm{Li}\right) n$ angular distribution measured in this work includes the contributions of the ground and first excited states in ${ }^{7} \mathrm{Li}$. The events of these two states cannot be separated because their energy difference is only $0.48 \mathrm{MeV}$ which is less than the energy spread $(0.62 \mathrm{MeV})$ of the ${ }^{6} \mathrm{He}$ beam.

The ${ }^{2} \mathrm{H}\left({ }^{6} \mathrm{He},{ }^{7} \mathrm{Li}\right) n$ reaction at the present energy is dominated by the direct process. In the angular range of $10^{\circ}-40^{\circ}$ where the differential cross-sections were used to extract the spectroscopic factor, the compound nucleus (CN) contribution was estimated to be $2 \%$ by using the nuclear-reaction program Talys [17], thus it was negligible in the following calculations. The ${ }^{2} \mathrm{H}\left({ }^{6} \mathrm{He},{ }^{7} \mathrm{Li}\right) n$ reaction leading to the ground state in ${ }^{7} \mathrm{Li}$ is a $\left(J=0^{+}, T=\right.$ $1) \rightarrow\left(J=3 / 2^{-}, T=1 / 2\right)$ transition. Parity and angularmomentum considerations dictate that only $1 p_{3 / 2}$ pickup is possible. The ${ }^{2} \mathrm{H}\left({ }^{6} \mathrm{He},{ }^{7} \mathrm{Li}^{*}\right) n$ reaction leading to the first excited state in ${ }^{7} \mathrm{Li}$ is a $\left(J=0^{+}, T=1\right) \rightarrow(J=$ $\left.1 / 2^{-}, T=1 / 2\right)$ transition and only the $1 p_{1 / 2}$ pickup contributes to the reaction. The relationship among the experimental differential cross-sections, the DWBA calculations and the spectroscopic factors can be expressed as

$$
\left(\frac{\mathrm{d} \sigma}{\mathrm{d} \Omega}\right)_{\exp }=S_{d} S^{7 \mathrm{Li}}\left(\frac{\mathrm{d} \sigma}{\mathrm{d} \Omega}\right)_{\mathrm{gs}}+S_{d} S{ }_{\mathrm{Li}^{*}}\left(\frac{\mathrm{d} \sigma}{\mathrm{d} \Omega}\right)_{\mathrm{ex} 1},
$$

where $\left(\frac{\mathrm{d} \sigma}{\mathrm{d} \Omega}\right)_{\exp }$ is the experimental differential crosssection, $\left(\frac{\mathrm{d} \sigma}{\mathrm{d} \Omega}\right)_{\mathrm{gs}}$ and $\left(\frac{\mathrm{d} \sigma}{\mathrm{d} \Omega}\right)_{\mathrm{ex} 1}$ are the calculational differential cross-sections for the ${ }^{2} \mathrm{H}\left({ }^{6} \mathrm{He},{ }^{7} \mathrm{Li}\right) n$ and ${ }^{2} \mathrm{H}\left({ }^{6} \mathrm{He},{ }^{7} \mathrm{Li}^{*}\right) n$ reactions. $S_{d}$ is the spectroscopic factor for $d \rightarrow p+n$, which was derived to be 0.859 from ref. [18] with the standard geometry parameters $r_{0}=1.25 \mathrm{fm}$ and $a=0.65 \mathrm{fm} . S_{7 \mathrm{Li}}$ and $S_{7} \mathrm{Li}^{*}$ are the proton spectroscopic factors of the ground and first excited states in ${ }^{7} \mathrm{Li}$. According to the translationally invariant shell model [19] calculation with the code DESNA [20] and Boyarkina's wave function tables [21], the ratio of $S_{7 \mathrm{Li}} / S_{7} \mathrm{Li}^{*}$ was found to be 1.0 [22]. Thus, the proton spectroscopic factors in ${ }^{7} \mathrm{Li}$ can be extracted through eq. (1) by normalizing DWBA calculations to the experimental data.

The code FRESCO [23] was used to compute the angular distribution of the ${ }^{2} \mathrm{H}\left({ }^{6} \mathrm{He},{ }^{7} \mathrm{Li}\right) n$ reaction leading to the ground and first excited states of ${ }^{7} \mathrm{Li}$. Five sets of ${ }^{6} \mathrm{He}+d$ potential parameters was utilized to extract the proton spectroscopic factor of ${ }^{7} \mathrm{Li}$. They are labeled as D1, D2, D3, D4 and D5, respectively, as listed in table 1 . Set D1 is a global deuteron optical model potential for the energies up to $183 \mathrm{MeV}$, based on the existing experimental data of elastic scattering angular distributions and nonelastic cross-sections for the target nuclei ranging from ${ }^{6} \mathrm{Li}$ to ${ }^{238} \mathrm{U}$ [24]. Set D2 is obtained from the analysis of an extensive set of data [25], which includes the results of both polarized and unpolarized elastic deuteron scattering on the nuclei from ${ }^{27} \mathrm{Al}$ to ${ }^{238} \mathrm{Th}$ in the energy range of $E_{d}=12-90 \mathrm{MeV}$. Recently, the expression for D2 has been extrapolated to the nuclei of $A<27$ [16]. Set D3 is based on the analysis of the elastic scattering of
Table 1. Optical potential parameters used in DWBA calculations, the depths are in $\mathrm{MeV}$, the geometry parameters in $\mathrm{fm}$, the geometrical parameters of single-particle bound state are set to be $r_{0}=1.25 \mathrm{fm}$ and $a=0.65 \mathrm{fm}$.

\begin{tabular}{ccccccc}
\hline Set No. & D1 & D2 & D3 & D4 & D5 & N1 \\
\hline$V$ & 89.56 & 86.32 & 76.41 & 86.80 & 80.53 & 45.27 \\
$r_{r}$ & 1.15 & 1.17 & 1.25 & 1.13 & 1.15 & 1.13 \\
$a_{r}$ & 0.74 & 0.73 & 0.77 & 0.80 & 0.81 & 0.69 \\
$W_{V}$ & 1.86 & 0.18 & & & & 2.12 \\
$r_{v}$ & 1.36 & 1.325 & & & & 1.11 \\
$a_{v}$ & 0.67 & 0.66 & & & & 0.69 \\
$W_{D}$ & 10.46 & 12.33 & 13.0 & 12.0 & 17.31 & 4.54 \\
$r_{s}$ & 1.42 & 1.325 & 1.25 & 1.56 & 1.34 & 1.11 \\
$a_{s}$ & 0.64 & 0.66 & 0.65 & 0.68 & 0.68 & 0.69 \\
$V_{s o}{ }$ & 3.56 & 6.98 & 6.0 & 5.2 & & 5.90 \\
$r_{s o}$ & 0.97 & 1.07 & 1.25 & 0.85 & & 0.71 \\
$a_{s o}$ & 1.01 & 0.66 & 0.76 & 0.48 & & 0.63 \\
$r_{c}$ & 1.30 & 1.30 & 1.30 & 1.30 & 1.15 & \\
Ref. & {$[24]$} & {$[25]$} & {$[26]$} & {$[27,28]$} & {$[29]$} & {$[34]$} \\
\hline
\end{tabular}

${ }^{b} V_{s o}$ for D1-D4 are multiplied by 0.5 in the calculation with FRESCO.

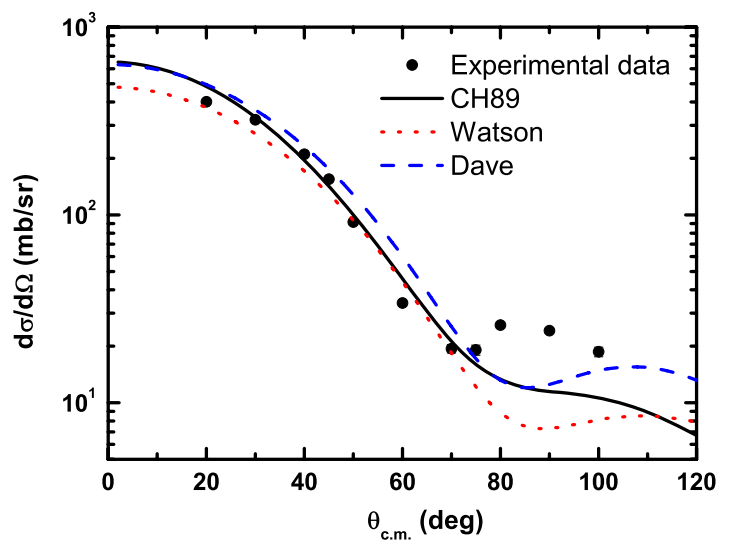

Fig. 4. Angular distribution of the ${ }^{7} \mathrm{Li}(n, n)^{7} \mathrm{Li}$ reaction at $E_{n}=14.5 \mathrm{MeV}$. The experimental data are taken from ref. [37].

$52 \mathrm{MeV}$ deuterons from 27 nuclei [26]. Set D4 is deduced from the elastic scattering of $30 \mathrm{MeV}$ polarized deuterons on 10 nuclei [27] and the energy dependence of $V$ [28] is adopted in the calculation. Set D5 is the deuteron global potential for the nuclei of $Z \geq 12$ with deuteron energies from 12 to $25 \mathrm{MeV}$ [29]. There are some nucleon-nucleus global optical-model potentials [29-34], in which the CH89 global optical potential model [34] was proved to give a better fitting to the experimental data [35] and have been successfully used in the DWBA calculations for the $(d, p)$ reaction on light nuclei $[3,16,36]$. We also tested the validity of the CH89 optical potential parameters by comparing the existing elastic scattering data of the neutron on ${ }^{7} \mathrm{Li}$ at $E_{n}=14.5 \mathrm{MeV}$ [37] with the calculated results using CH89 [34] and other two sets of optical potential for $1 p$ shell nuclei $[30,33]$. As shown in fig. 4, the CH89 optical potential gives the best fitting to the ${ }^{7} \mathrm{Li}(n, n)^{7} \mathrm{Li}$ angular distribution, and thus is chosen in the calculations. The 


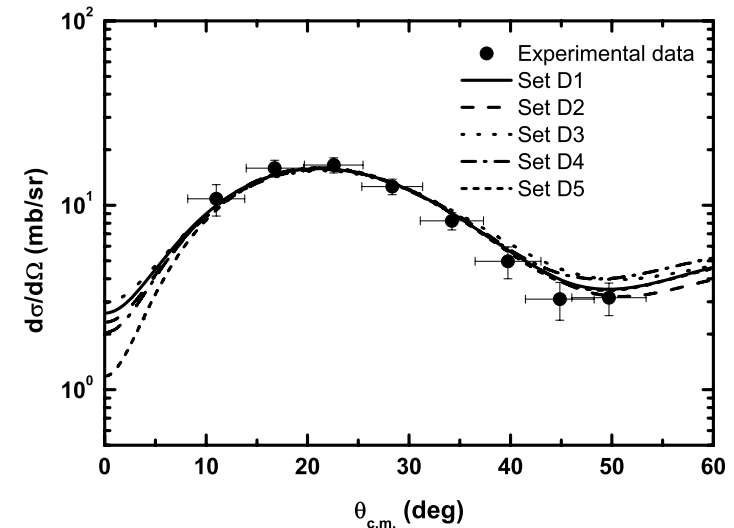

Fig. 5. Comparison of the experimental angular distribution for ${ }^{2} \mathrm{H}\left({ }^{6} \mathrm{He},{ }^{7} \mathrm{Li}\right) n$ with DWBA calculations using 5 sets of optical potential parameters.

potential parameters of the exit channel are labeled as N1 in table 1 .

The normalized angular distributions calculated with the above-mentioned optical potentials are presented in fig. 5 together with the experimental data. One can see that the first peak of the experimental angular distribution is fairly reproduced by all the optical potentials. The extracted spectroscopic factors with five data points in the peak are $0.40,0.38,0.36,0.48$ and 0.47 , respectively. Their average is 0.42 with a standard deviation of 0.05 .

In order to estimate the uncertainty of the spectroscopic factor from the potential of ${ }^{6} \mathrm{He}+p$ bound state in ${ }^{7} \mathrm{Li}$, we have tested the influence of the geometrical parameters $\left(r_{0}\right.$ and $\left.a\right)$ on the spectroscopic factor. The radius was changed from 1.10 to $1.40 \mathrm{fm}$ while the diffuseness was adjusted to reproduce the rms radius of the valence proton in ${ }^{7} \mathrm{Li}$ which was calculated with the charge rms radii of ${ }^{6} \mathrm{He}$ and ${ }^{7} \mathrm{Li}$ reported in refs. $[38,39]$ according to

$$
r_{\mathrm{Li}}^{2}=\frac{1}{Z+1}\left(Z r_{{ }_{\mathrm{H}} \mathrm{He}}^{2}+r_{p}^{2}+\frac{Z}{Z+1} r_{v}^{2}\right),
$$

where $r_{7 \mathrm{Li}}, r_{6} \mathrm{He}$ and $r_{p}$ are the charge rms radii for ${ }^{7} \mathrm{Li}$, ${ }^{6} \mathrm{He}$ and proton, respectively. $r_{v}$ is the rms radius of the valence proton orbit in ${ }^{7} \mathrm{Li}, Z$ denotes the proton number in ${ }^{6} \mathrm{He}$. The above changes led to a $3 \%$ uncertainty of the deduced spectroscopic factor, which was negligible compared with the uncertainty from deuteron- ${ }^{6} \mathrm{He}$ potential parameters. The final value of the proton spectroscopic factor of ${ }^{7} \mathrm{Li}$ was extracted to be $0.42 \pm 0.06$. The error was from the measurement $(5 \%)$ and the uncertainties of optical potential parameters $(12 \%)$.

Figure 6 shows the comparison of ${ }^{7} \mathrm{Li}$ spectroscopic factors from theoretical calculations and experiments. The ${ }^{7} \mathrm{Li}$ spectroscopic factor obtained in our work is smaller than the theoretical calculations reported in refs. [12] and [40]. Comparing with the experimental results, ours is $34 \%$ smaller than that extracted from the ${ }^{7} \mathrm{Li}(n, d){ }^{6} \mathrm{He}$ reaction [13], and in good agreement with that from the ${ }^{7} \mathrm{Li}\left(e, e^{\prime} p\right)$ reaction by Lapikás et al. [14].

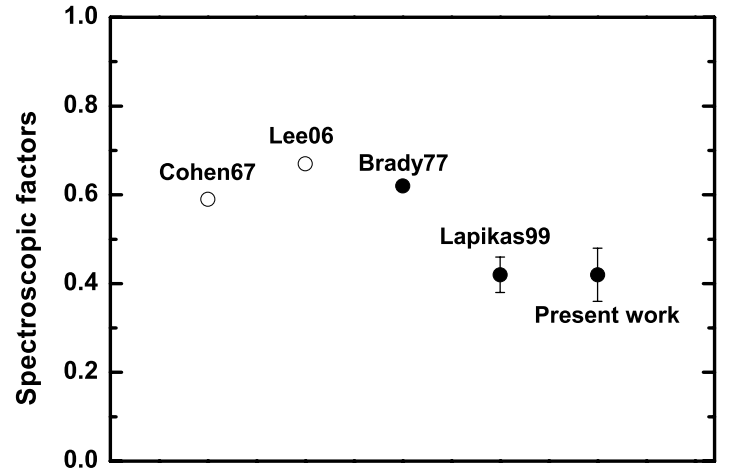

Fig. 6. Comparison of spectroscopic factors for ${ }^{7} \mathrm{Li}\left(3 / 2^{-}\right) \rightarrow$ ${ }^{6} \mathrm{He}+p$. The solid and open circles represent the experimental and theoretical results, respectively.

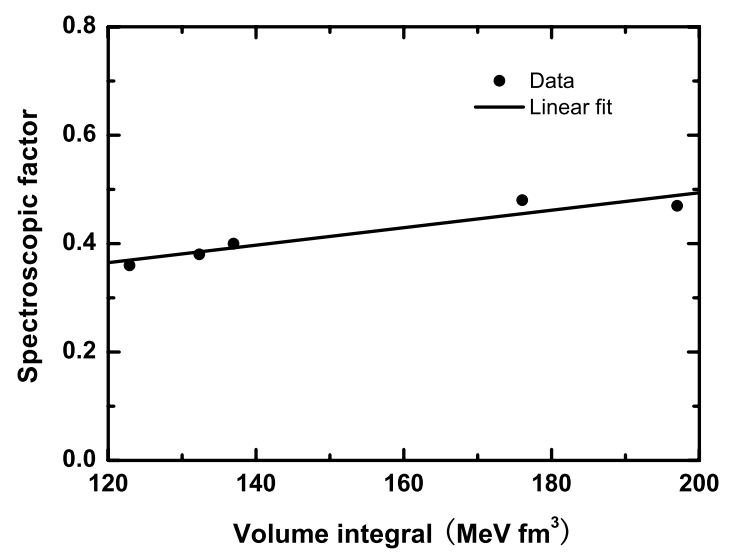

Fig. 7. Spectroscopic factors as a function of the volume integral for the imaginary part of the optical potentials. The solid line is a linear fit of the data points.

\section{Conclusion and discussion}

The ${ }^{2} \mathrm{H}\left({ }^{6} \mathrm{He},{ }^{7} \mathrm{Li}\right) n$ angular distribution was measured at $E_{6 \mathrm{He}}=36.4 \mathrm{MeV}$ using the secondary beam facility of the HI-13 tandem accelerator in Beijing. The proton spectroscopic factor in the ${ }^{7} \mathrm{Li}$ ground state is extracted to be $0.42 \pm 0.06$. The merit of ${ }^{2} \mathrm{H}\left({ }^{6} \mathrm{He},{ }^{7} \mathrm{Li}\right) n$ is that the reaction product is relatively clean as compared to the ${ }^{2} \mathrm{H}\left({ }^{7} \mathrm{Li},{ }^{3} \mathrm{He}\right){ }^{6} \mathrm{He}$ reaction in which there are several reaction channels (such as breakup and three body) leading to ${ }^{6} \mathrm{He}$ and coincidence measurement is needed.

The error $(14 \%)$ of the spectroscopic factor given in this work mainly arose from the uncertainty $(12 \%)$ of the optical potentials. Thus, it is important to further investigate the influence of optical potentials. It is found that the volume integrals for the real part of five sets potentials only differ by a factor of $3 \%$, while those for the imaginary part deviate up to $20 \%$. The uncertainty of the spectroscopic factor mainly arises from the uncertainty of the imaginary optical potentials. Figure 7 shows a linear relationship between the volume integrals of the imaginary part and the spectroscopic factors. Therefore, the uncertainty of the extracted spectroscopic factors might be reduced by determining the imaginary volume inte- 
gral for the deuteron-nucleus precisely. Generally speaking, the angular distribution of the elastic scattering can provide fairly good information on the real part of the optical potential. However, it can only give relatively poor information on the imaginary part of the optical potential. Consequently, it is of importance for extracting the imaginary potential parameters to study the deuteron-nucleus reactions besides elastic scattering.

We also extracted the proton spectroscopic factor in ${ }^{7} \mathrm{Li}$ with the adiabatic deuteron potentials [41,42] constructed by CH89 nucleon potentials [34]. The value is 0.41 , close to the average of the spectroscopic factors extracted with the optical potentials (D1-D5) in table 1 . The reason could be that the depths of the adiabatic deuteron potentials were adjusted so that the volume integral of $U_{d}$ equaled that of $\left(U_{n}+U_{p}\right)$, and the volume integrals for the optical potential of nucleon-nucleus are usually studied well as compared to that of deuteron-nucleus. This may provide an alternative way to constrain the deuteronnucleus optical potential.

The authors would like to thank Prof. I.J. Thompson for his help on the theoretical calculations. This work is supported by the National Basic Research Programme of China under Grant No. 2007CB815003, the National Natural Science Foundation of China under Grant Nos. 10675173, 10705053, 10735100 and 10975193

Open Access This article is distributed under the terms of the Creative Commons Attribution Noncommercial License which permits any noncommercial use, distribution, and reproduction in any medium, provided the original author(s) and source are credited.

\section{References}

1. M.H. Macfarlane, J.B. French, Rev. Mod. Phys. 32, 567 (1960).

2. M. Assunção, R. Lichtenthäler, V. Guimarães, A. LépineSzily, G.F. Lima, A.M. Moro, Phys. Rev. C 70, 054601 (2004).

3. M.B. Tsang, Jenny Lee, W.G. Lynch, Phys. Rev. Lett. 95 , 222501 (2005).

4. Z.H. Li, W.P. Liu, X.X. Bai, B. Guo, G. Lian, S.Q. Yan, B.X. Wang, S. Zeng, Y. Lu, J. Su, Y.S. Chen, K.S. Wu, N.C. Shu, T. Kajino, Phy. Rev. C 71, 052801 (2005).

5. A.H. Wuosmaa, K.E. Rehm, J.P. Greene, D.J. Henderson, R.V.F. Janssens, C.L. Jiang, L. Jisonna, E.F. Moore, R.C. Pardo, M. Paul, D. Peterson, S.C. Pieper, G. Savard, J.P. Schiffer, R.E. Segel, S. Sinha, X. Tang, R.B. Wiringa, Phys. Rev. Lett. 94, 082502 (2005).

6. Z.H. Li, B. Guo, S.Q. Yan, G. Lian, X.X. Bai, Y.B. Wang, S. Zeng, J. Su, B.X. Wang, W.P. Liu, N.C. Shu, Y.S. Chen, H.W. Chang, L.Y. Jiang, Phys. Rev. C 74, 035801 (2006).

7. K.W. Kemper, R.L. White, L.A. Charlton, G.D. Gunn, G.E. Moore, Phys. Lett. B 52, 179 (1974).

8. R.L. White, K.W. Kemper, Phys. Rev. C 10, 1372 (1974).

9. J.E. Kim, W.W. Daehnick, Phys. Rev. C 23, 742 (1981).

10. K.W. Kemper, G.E. Moore, R.J. Puigh, R.L. White, Phys. Rev. C 15, 1726 (1977).
11. M.E. Williams-Norton, G.M. Hudson, K.W. Kemper, G.E. Moore, G.A. Norton, R.J. Puigh, A.F. Zeller, Phys. Rev. C 12, 1899 (1975).

12. S. Cohen, D. Kurath, Nucl. Phys. A 101, 1 (1967).

13. F.P. Brady, N.S. King, B.E. Bonner, M.W. McNaughton, J.C. Wang, W.W. True, Phys. Rev. C 16, 31 (1977).

14. L. Lapikás, J. Wesseling, R.B. Wiringa, Phys. Rev. Lett. 82, 4404 (1999).

15. X. Bai, W. Liu, J. Qin, Z. Li, S. Zhou, A. Li, Y. Wang, Y. Cheng, W. Zhao, Nucl. Phys. A 588, 273c (1995).

16. X.D. Liu, M.A. Famiano, W.G. Lynch, M.B. Tsang, J.A. Tostevin, Phys. Rev. C 69, 064313 (2004).

17. A.J. Koning, M.C. Duijvestijn, Nucl. Instrum. Methods: Phys. Res. B 248, 197 (2006).

18. L.D. Blokhintsev, I. Borbely, E.I. Dolinskii, Sov. J. Part. Nucl. 8, 485 (1977).

19. Yu.F. Smirnov, Yu.M. Tchuvil'sky, Phys. Rev. C 15, 84 (1977).

20. A.T. Rudchik, Yu.M. Tchuvil'sky, Ukr. J. Phys. 30, 819 (1985).

21. A.N. Boyarkina, Structure of $1 p$-shell nuclei, Moscow State University report (1973).

22. A.A. Rudchik, A.T. Rudchik, G.M. Kozeratska, O.A. Ponkratenko, E.I. Koshchy, A. Budzanowski, B. Czech, S. Kliczewski, R. Siudak, I. Skwirczyńska, A. Szczurek, S.Yu. Mezhevych, K.W. Kemper, J. Choiński, T. Czosnyka, L. Glowacka, Phys. Rev. C 72, 034608 (2005).

23. I.J. Thompson, Comput. Phys. Rep. 7, 167 (1988).

24. Haixia An, Chonghai Cai, Phys. Rev. C 73, 054605 (2006).

25. W.W. Daehnick, J.D. Childs, Z. Vrcelj, Phys. Rev. C 21, 2253 (1980).

26. F. Hinterberger, G. Mairle, U. Schmidt-Rohr, G.J. Wagner, P. Turek, Nucl. Phys. A 111, 265 (1968).

27. G. Perrin, Nguyen Van Sen, J. Arvieux, R. Darves-Blanc, J.L. Durand, A. Fiore, J.C. Gondrand, F. Merchez, C. Perrin, Nucl. Phys. A 282, 221 (1977).

28. J. Vernotte, G. Berrier-Ronsin, J. Kalifa, R. Tamisier, B.H. Wildenthal, Nucl. Phys. A 571, 1 (1994).

29. C.M. Perey, F.G. Perey, At. Data Nucl. Data Tables 17, 1 (1976).

30. B.A. Watson, P.P. Singh, R.E. Segel, Phys. Rev. 182, 977 (1969).

31. F.D. Bechetti jr., G.W. Greenlees, Phys. Rev. 182, 1190 (1969).

32. J.J. Menet, E.E. Gross, J.J. Malanify, A. Zucker, Phys. Rev. C 4, 1114 (1971).

33. J.H. Dave, C.R. Gould, Phys. Rev. C 28, 2212 (1983).

34. R.L. Varner, W.J. Thompson, T.L. McAbee, E.J. Ludwig, T.B. Clegg, Phys. Rep. 201, 57 (1991).

35. X.D. Liu, PhD Thesis, Michigan State University (2005).

36. B. Guo, Z.H. Li, W.P. Liu, X.X. Bai, J. Phys. G: Nucl. Part. Phys. 34, 103 (2007).

37. R.J. Danahy, PhD Thesis, Worcester Polytechnic Institute (1980).

38. I. Tanihata, H. Hamagaki, O. Hashimoto, Y. Shida, N. Yoshikawa, K. Sugimoto, O. Yamakawa, T. Kobayashi, N. Takahashi, Phys. Rev. Lett. 55, 2676 (1985).

39. C. Forssén, E. Caurier, P. Navrátil, Phys. Rev. C 79, 021303(R) (2009).

40. J. Lee, J.A. Tostevin, B.A. Brown, F. Delaunay, W.G. Lynch, M.J. Saelim, M.B. Tsang, Phys. Rev. C 73, 044608 (2006).

41. G.R. Satchler, Phys. Rev. C 4, 1485 (1971).

42. G.L. Wales, R.C. Johnson, Nucl. Phys. A 274, 168 (1976). 